\title{
“SI HUBIERA SOBREVIVIDO EN PRAGA, ÁMSTERDAM O LODZ...” LAS POLÍTICAS DE LOCALIZACIÓN EN EL PENSAMIENTO DE ADRIENNE RICH ${ }^{1}$
}

\author{
MARIAN PÉREZ BERNAL \\ Universidad Pablo de Olavide
}

\begin{abstract}
En este artículo reflexionamos sobre el concepto "políticas de localización" de Adrienne Rich, que consideramos muy fructífero para pensar la identidad. Partimos del concepto de experiencia de Rich con sumo cuidado de evitar caer en discursos esencialistas de la identidad y teniendo en todo momento presente la importancia del lenguaje y de la narración en la construcción de la identidad. Las políticas de localización nos permiten ver nuestra identidad como una intersección de múltiples hilos que construye la trama cambiante, rica y en ocasiones confusa de nuestra identidad. Tomar conciencia de esto puede ser de ayuda para entablar el diálogo, construir experiencias compartidas y establecer alianzas entre las diferentes corrientes del feminismo.
\end{abstract}

PALABRAS CLAVE: feminismo, identidad, experiencia, políticas de localización.

"If I had survived in Prague, Amsterdam, or Lodz..." Politics of Location in Adrienne Rich's Thought

In this article, we reflect on Adrienne Rich's concept of the "politics of location", which we deem very useful when thinking about identity. We begin with Rich's concept of experience, taking great care not to lapse into essentialist discourses of identity and always keeping in mind the importance of language and narration in the construction of identity. A politics of location allows us to see our identity as an intersection of multiple threads that weave the changing, rich, and sometimes confusing fabric of our identity. To develop an awareness thereof can help to initiate dialogue, build shared experiences, and establish alliances between the different currents of feminism.

KEY WORDS: feminism, identity, experience, politics of location.

Una mujer que piensa duerme con monstruos. -ADRIENNE RICH, Instantáneas de una nuera (1963)

\footnotetext{
${ }^{1}$ Este trabajo ha sido realizado gracias a la financiación del proyecto de investigación "El desván de la razón: Cultivo de las pasiones, identidades éticas y sociedades digitales" (FFI2017-82535-P: PAIDESOC).
}

Pérez Bernal, Marian (2021), “'Si hubiera sobrevivido en Praga, Ámsterdam o Lodz...' Las políticas de localización en el pensamiento de Adrienne Rich", Lectora, 27: 291-307. ISSN: 1136-5781 D.O.I.: 10.1344/Lectora2021.27.15, mdperber@upo.es

Recepció: 14 de desembre de 2020 - Acceptació: 21 d'abril de 2021 
En el artículo de 1984 "Notes towards a Politics of Location", Adrienne Rich acuña el concepto de "políticas de localización”. Este enfoque está sin duda en deuda con los análisis de las feministas chicanas y negras. Por un lado, Gloria Anzaldúa y Cherríe Moraga (1981), y por otro Gloria Hull, Patricia Bell Scott y Barbara Smith (1982) fueron las primeras que sacaron a la luz los análisis y las posiciones políticas de las feministas de color señalando que el feminismo blanco no había tenido en cuenta el clasismo, el racismo, el colonialismo, el imperialismo y el heterosexismo (De Lauretis, 1987: 10). Es en este contexto del feminismo de los ochenta donde se comienza a desafiar el sujeto normativo del feminismo occidental, en el que se plantea la política de localización como una estrategia discursiva de las feministas radicales lesbianas para explicar el carácter situado del conocimiento y para formular nuevas formas de subjetividad capaces de dar respuesta a las reivindicaciones realizadas desde el feminismo negro.

Con las políticas de la localización, Rich abrió la puerta a un tema nuevo, defendido también por autoras como Teresa de Lauretis, Rosi Braidotti o Linda Alcoff. Las políticas de localización serían en cierto modo equivalentes a las "posiciones de ubicación" de Chandra Mohanty (1987), ${ }^{2}$ al "conocimiento situado" de Donna Haraway (1988) y a las "posiciones del sujeto" de Chantal Mouffe (1999). Las políticas de localización comparten con las teorías feministas del punto de vista presupuestos sobre el carácter situado tanto del sujeto como del conocimiento; para ambas las verdades están mediadas por la ubicación material e histórica de un sujeto en el mundo. Si bien establecer comparaciones entre estos enfoques abre sendas de reflexión muy interesantes, por cuestiones de tiempo y de espacio no son por las que caminaremos ahora. Con todo, no queremos dejar de señalar una diferencia importante apuntada por Fox y que comparto:

Instead, I conceive of standpoint theory and a politics of location in a relationship similar to that of two overlapping circles. That is, the two share a set of assumptions, but also distinctive characteristics of each. Feminist standpoint theory is distinguished by its greater emphasis on epistemology -on issues of how knowledge is constructed. A politics of location is distinguished by its focus on discursive representations of

\footnotetext{
${ }^{2}$ En "Feminist Encounters: Locating the Politics of Experience", Mohanty (1987) reconoce su deuda con las políticas de localización y titula con un verso de Rich, "A Place on the Map is Also a Place in History”, uno de los epígrafes. Mohanty (1987: 30) define las políticas de localización como los límites históricos, geográficos, culturales, psíquicos e imaginativos que proporcionan el terreno para la definición política y la autodefinición de las feministas estadounidenses contemporáneas.
} 
identity positions - on how subjectivity is discursively deconstructed and then reconstructed. (Fox, 2004: 46)

Consideramos que la política de localización puede ser un concepto muy fructífero tanto para pensar la identidad como para plantear alianzas entre las diferentes corrientes dentro del feminismo. Pensar la identidad desde este concepto puede ayudar a evitar caer en políticas identitarias obsoletas y, al mismo tiempo, conseguir establecer nuevas formas de subjetividad capaces de crear nuevas coaliciones. La expresión "políticas de localización" supone un esfuerzo por parte de Rich de alejarse de los planteamientos de un feminismo occidental hegemónico que universaliza las experiencias de las mujeres blancas, heterosexuales, occidentales y de clase media, construyendo así un sujeto del feminismo normativo y, por ello mismo, limitante. Rich articuló las políticas de la localización como un antídoto contra esto. Desde su perspectiva, no podemos hablar de una "cuestión femenina" que nos afecta a todas por igual, ya que cada mujer está situada siempre en un lugar y en un tiempo, y esta localización afecta sus experiencias y su forma de mirar la realidad. En "Es la lesbiana que hay en nosotras", afirma:

Si concebimos al feminismo como algo más que una etiqueta frívola, si lo concebimos como una ética, una metodología, una manera más compleja de pensar sobre, y por tanto de mayor responsabilidad al incidir en las condiciones de la vida humana, necesitaremos un autoconocimiento que solamente puede desarrollarse a través de una continua atención apasionada a todas las experiencias femeninas. No puedo imaginar una evolución feminista que nos conduzca a cambios radicales en los campos de lo privado y de lo político que no esté enraizada en la convicción de que todas las vidas de las mujeres son importantes y que las vidas de los hombres no pueden ser entendidas enterrando las vidas de las mujeres. (Rich, 2010d: 310-311)

Políticas de localización y experiencias van de la mano. Ya Scott nos advertía del peligro del término "experiencia" por la facilidad de que nos lleve a enfoques esencializadores de la identidad. Evitar esa deriva no nos puede hacer renunciar a un término de gran potencial. En la línea de Scott, abogamos por utilizarlo teniendo presente esos peligros y, si es necesario, redefiniendo su significado (1991: 797). Cuando se habla de reinterpretar el conocimiento a la luz de la experiencia hay que tener presente la diversidad de experiencias vividas por las mujeres.

En este punto, Rich cita a Virginia Woolf: "As a woman I have no country. As a woman I want no country. As a woman my country is the whole world" (1993: 234). En 2018, diez temporeras marroquíes que trabajaban en la recogida de la fresa en Huelva acudieron a la Audiencia Nacional para denunciar por delitos de 
lesa humanidad y trata de seres humanos a los empresarios que las habían contratado. Las trabajadoras, mujeres rurales con hijos menores a su cargo y en situación de especial vulnerabilidad, denunciaron las penosas condiciones de trabajo y de vida a las que supuestamente fueron sometidas. También manifestaron haber sido objeto de acoso sexual. No era la primera vez que esto sucedía. Ya hay sentencia firme por denuncias similares en Huelva en 2014. ¿Podemos pensar en esas temporeras marroquíes diciendo que son ciudadanas del mundo? Su país no es el mundo entero y por supuesto no es España. El lugar donde nacieron condiciona el discurso que pueden construir. Para algunas es más fácil que para otras ser ciudadanas del mundo y el feminismo debe ocuparse siempre de los eslabones más débiles de la cadena porque de ellos depende que la cadena no se rompa.

Aun entendiendo la grandeza del mensaje que Woolf pretendía trasmitir, Rich le da la vuelta poniendo en cuestión la viabilidad de esa idea tan bien intencionada, recordándole que las cosas no son tan fáciles. Por mucho que quiera, no puedo despojarme del país al que pertenezco. Por mucho que reniegue y condene al gobierno de mi país - dirá Rich—, llego a los sitios como ciudadana estadounidense y eso está pesando sobre mí. Un lugar sobre el mapa es también un lugar en la historia. Pesa en la vida de las temporeras marroquíes que van a Huelva y pesó en la vida de Rich:

Si hubiera sobrevivido en Praga, Ámsterdam o Lodz y en las estaciones de tren que eran los puntos de deportación, sería otra. Mi lugar, quizás Oriente Medio o Latinoamérica, mi idioma, otro. O podría no estar en ningún cuerpo en absoluto. Pero yo soy una judía norteamericana que nació y creció a tres mil millas de la guerra de Europa. (Rich, 1994a: 216)

Lejos de ser una tabula rasa, el cuerpo ya está sujeto antes del nacimiento a una variedad de significados culturales (Eagleton, 2000: 304). Rich era mischling. Este término fue usado en las leyes raciales de la Alemania nazi para referirse a las personas que tenían uno o dos abuelos judíos. Rich nació el 16 de mayo de 1929. Tenía cuatro años al comienzo del Tercer Reich. Era una persona lo suficientemente judía como para que la ubicación geográfica hubiera desempeñado sobre ella un papel determinante (Rich, 1994a: 216). ${ }^{3}$ Si no hubiera nacido en Baltimore sino en Praga, Lódz o Ámsterdam, su vida habría sido muy diferente. Ana Frank nació en Ámsterdam ese mismo año. Esto nos recuerda la importancia de las políticas de localización. La geografía la salvó. Esa misma geografía fue en parte la responsable de que se casara a los veinte y tuviera tres hijos antes de los treinta.

\footnotetext{
${ }^{3}$ Curiosamente sería considerada judía por los antijudíos y no judía por los judíos al no ser su madre judía.
} 
Eran los años cincuenta y las mujeres de clase media tenían como meta la perfección doméstica (Rich, 1972: 22).

\section{Las intersecciones que nos conforman}

No creo que sea casual que Rich se interesara por estas cuestiones. Su propia vida es un ejemplo paradigmático de la importancia que tienen las políticas de localización a la hora de construir la identidad.

Tribal loyalties aside, and even if nation-states are now just pretexts used by multinational conglomerate to serve their interests, I need to understand how a place on the map is also a place in history within which as a woman, a Jew, a lesbian, a feminist I am created and trying to create. (Rich, 1994a: 212)

Esta mujer judía, lesbiana y feminista nos recuerda que nació en un hospital donde las mujeres blancas y las negras estaban separadas a la hora de parir. "Fui definida como blanca, antes de ser definida como mujer" (Rich, 1994a: 215). Estamos situadas en múltiples sentidos. Las localizaciones se ven interconectadas con patrones complejos $\mathrm{y}$, aunque sean determinadas localizaciones las que pueden estar en primer plano en momentos concretos, siempre hay toda una gama de factores determinantes por detrás también actuando. En aquel contexto era más importante el color de la piel que el sexo. Igual que en la ex-Yugoslavia, por ejemplo, durante la guerra el nacionalismo mató la diferencia sexual (Papic, 1994). Allí una era serbia, croata o bosnia; no era posible ser solo una mujer (Braidotti, 2004: 78). Vemos así que la identidad no es una entidad fija sino que se halla en construcción - la experiencia y la identidad deben pensarse en perspectiva histórica- e influyen en ella los relatos que contamos. Era tan fuerte en ese contexto en concreto lo que significaba ser serbia o bosnia que el hecho de ser mujer se difuminaba, igual que pesaba más ser blanca o negra que mujer u hombre en Baltimore cuando nació Rich. Lo que constituye una ubicación cambia y se reforma constantemente, mientras que las ubicaciones anteriores se pueden recordar y reconstruir de diferentes maneras (Eagleton, 2000: 300). Nos encontramos en todo momento en la tensión entre lo ya construido y lo aún por construir, ya que las políticas de localización incluyen la potencialidad de reescribir las subjetividades de acuerdo con las nuevas narrativas de las experiencias de las mujeres (Fox, 2004: 47).

Rich es muy consciente de las intersecciones que nos conforman y hasta qué punto debemos tenerlas presentes para analizar la realidad. Podría ser considerada como un paradigma de la interseccionalidad antes de que Crenshaw (1989) acuñara este concepto. Con este término se apunta a cómo el género, la etnicidad, la sexualidad, la clase, la diversidad funcional, la edad o el nivel de formación, entre 
otros, son ejes de categorización y diferenciación, están imbricados de manera compleja en los procesos de dominación. ${ }^{4}$ Cuando Rich se pregunta por qué es tan importante ser consciente de las políticas de localización, responde, citando a Paulo Freire, que solo las personas conscientes de que se hallan determinadas por sus circunstancias y que reflexionan sobre ello podrán liberarse de las mismas (2010a: 100). Su punto de vista personal ha sido creado por las condiciones políticas. No es un hombre y es blanca en una sociedad supremacista; ha sido educada desde la perspectiva de una clase determinada, su padre era un judío asimilado en un mundo antijudío y su madre era una mujer protestante del sur (Rich, 1983: 524525). Todos estos elementos han configurado su subjetividad múltiple y compleja. También en su yo poético se entrelazarán todas estas encrucijadas. Para Rich " $t]$ he self is selves" (Perreault, 1995: 32).

Su pertenencia al judaísmo por parte de su padre será para ella un tema central. En un poema de 1960, "Readings of History", dice de sí misma: "Dividida desde la raíz, ni gentil ni judía”. Sobre esta cuestión se centra en "Split at the root", que escribe porque necesita reclamar a su padre judío "rompiendo su silencio, sus tabúes" (Rich, 1982: 73). ¿Qué significa para ella ser judía?: "But what did it mean to be a Jewish lesbian? What did it mean to feel myself, as I did, both anti-Semite and Jew? And, as a feminist, how was I charting for myself the oppressions within the oppression?” (Rich, 1982: 89). Más adelante volveremos sobre estas marañas de opresiones.

No es casual qué se ve y qué se invisibiliza en estas identidades hechas de fragmentos. A pesar de que Rich había declarado su lesbianismo, sentía que muchas personas preferían no darse por enterados y lo obviaban. Este silencio tiene consecuencias y frente a ellas se rebela diciendo "[...] that invisibility is not just a matter of being told to keep your private life private, it's the attempt to fragment you, to prevent you from integrating, love and work and feeling and ideas, with the empowerment that that can bring" (Rich, 1994b: 200). Cualquier identidad que valga la pena alcanzar implica lucha y resistencia.

En tanto que el ser humano está siempre localizado, no hay una cuestión femenina que nos afecte a todas por igual. De ahí que afirme:

\footnotetext{
${ }^{4}$ Considero que hay una diferencia interesante entre la política de localización y la interseccionalidad de Crenshaw, en tanto que Rich no plantea una dicotomía fija entre la franja del privilegio y la franja de la opresión/resistencia, ya que puede cambiar de acuerdo con la situación. Ser judía te situaba en una situación de opresión en la Alemania nazi, pero te puede colocar en una situación de privilegio en los territorios ocupados. En este sentido, son interesantes las propuestas que abogan por pensar una interseccionalidad situada donde sea el contexto el que nos permita establecer qué ejes de opresión están operando y qué estrategias de resistencia pueden surgir en cada momento (Falcón, 2012).
} 
Nosotras rechazamos los enunciados que comienzan como "Las mujeres tienen siempre un instinto maternal" o "las mujeres siempre y en todo lugar son subyugadas por los hombres". Si hemos aprendido algo en estos años de feminismo de finales del siglo Xx es que ese "siempre" oculta lo que nosotras realmente necesitamos saber: cuándo, dónde y bajo qué condiciones resulta esto cierto. (Rich, 1994a: 214)

La esterilización fue uno de los problemas que le hizo comprender hasta qué punto raza y clase hacen diferentes hasta las experiencias de reproducción:

Como mujer blanca, de clase media y educada, que a fines de los años cincuenta tuve que rogar y argumentar para conseguir la esterilización después de haber tenido tres hijos, al principio sólo entendí que la esterilización a pedido era tan necesaria como el aborto legal y gratuito. Recuerdo vívidamente el impacto de la contradicción que surgió en los setenta: mientras el establishment médico estaba poco dispuesto a esterilizar a mujeres como yo, los mismos profesionales y el gobierno federal ejercían presión y coerción para esterilizar a gran número de mujeres indias, negras, chicanas, blancas pobres y portorriqueñas. (Rich, 1986: 38)

En aquel momento muchas feministas blancas no veían cómo la esterilización voluntaria podía convertirse en un arma de doble filo para las mujeres negras, para las indígenas, para las que vivían de ayudas del estado o para las menos inteligentes. La experiencia propia le dificultó ver qué podrían suponer esas políticas para la vida de otras mujeres. El problema de la esterilización le demostró cómo la raza y la clase diferencian incluso las experiencias más básicas, comunes a todas las mujeres (Rich, 1986: 39-40). Las condiciones a las que estaban sujetas unas mujeres y otras eran muy diferentes y eso llevaba aparejado que el discurso de la esterilización se leyera y tuviera condiciones muy diferentes en cada caso. Ya Scott (1991: 793) señaló cómo pueden existir conflictos entre los distintos sistemas discursivos.

Considerar al patriarcado un producto puro, no relacionado con la opresión económica o racial, nos ofrecería una visión sesgada de la realidad (Rich, 1986: 29). A menudo nos resulta complicado separar las opresiones vinculadas con la raza, la clase y el género porque en realidad en nuestra vida suelen aparecer de forma simultánea, de ahí que Rich hable de una "maraña [tangle] de opresiones" (1994a: 218). Da la sensación de que desenredar ese enredo es un trabajo sin fin. Siempre podríamos ir incluyendo nuevos matices, nuevos rasgos en esa localización que dibujarían de una forma nueva la subjetividad. De ahí que afirme: "there were particular historical currents on which my consciousness would come together, piece by piece" (Rich, 1983: 525). 
Las cuestiones que las mujeres plantean se presentan siempre desde un lugar y un tiempo, en una localización. El color de la piel, la clase, la religión, el espacio geográfico o el momento histórico en que uno vive interseccionan con la opresión de género en un contexto en que la subjetividad está sometida a permanente cambio. ${ }^{5}$ Además, una misma faceta puede, al mismo tiempo, ser positiva y negativa. Recuerda cómo tuvo la gran suerte de nacer en una casa llena de libros, con un padre que la animaba a leer y escribir, pero cómo eso también tuvo un coste. Durante mucho tiempo trató de no disgustar a ese padre (Rich, 1972: 21).

"La cuestión es desde dónde lo miramos" es el último verso del poema "Un atlas del mundo difícil, II" de Rich. Conocerse y nombrarse es ubicarse en distintas categorías como pueden ser judía, lesbiana, blanca, estadounidense y tras ello analizar dónde cada una de esas categorías la sitúan respecto a los grupos dominantes y los grupos marginales. La lucha por la identidad, si estamos en el centro de la sociedad, es muy diferente a si estamos en los márgenes. Analizar dónde estas categorías la sitúan la ayuda a conocerse a sí misma y en sus ensayos explora las epistemologías de la individualidad permitidas y requeridas, así como también las reprimidas. Esas fuerzas dividen a las mujeres entre sí y dentro de ellas (Perreault, 1995: 36).

\section{La reivindicación del propio cuerpo}

Desde la forma situada de entender al sujeto de Rich, se considera que la localización o situación más importante es el arraigo en el marco espacial del propio cuerpo: "Begin though, not with a continent or a country or a house, but with the geography closest in - the body" (Rich, 1994a: 211). Rich nos recuerda que una de las críticas del feminismo a la teoría marxista se relaciona con esto. Si bien el análisis de la situación marxista es fundamental, no es suficiente para analizar los problemas del feminismo. Cuando las personas luchan contra cualquier forma de sujeción, no podemos dejar de tener presente que la sujeción más específica de las mujeres es su incardinación en un cuerpo femenino (Rich, 1994a: 214). Frente a aquellos discursos que tratan de trascender lo corporal, ella lo reivindica, pero en lugar de hablar de "el cuerpo", prefiere hablar de "mi cuerpo", mucho menos grandioso pero por eso mismo mucho más apegado a la realidad. Su cuerpo con sus cicatrices, con sus heridas, con el estado de sus dientes y con la cantidad de calcio

\footnotetext{
${ }^{5}$ Judith Butler señala que las teorías feministas de la identidad, tras señalar predicados como etnicidad, clase o sexualidad, suelen acabar con un etcétera. Ese etcétera ilimitado sería el punto de partida para el pensamiento político feminista (Butler, 2007: 279). Las políticas de localización permiten explicar qué rasgos son significativos en cada situación. En un contexto, la religión ocupará un lugar central y la etnia pasaría al grupo de los etcéteras, y en otro será a la inversa. Hemos observado esto en la propia vida de Rich.
}

298

Lectora, 27 (2021): 291-307. ISSN: 1136-5781 D.O.I.: 10.1344/Lectora2021.27.15 
de sus huesos. Se trata de reconectar nuestro pensamiento y discurso con el cuerpo de ese ser humano particular e individual que es una mujer (Rich, 1994a: 213-215). El cuerpo dice mucho. Su cuerpo ha estado bien nutrido desde antes de que naciera y el estado de sus dientes, propio de una persona que se hacía dos revisiones al año, nos recuerda que se trata de una mujer de clase media.

Entramos ahora en un campo que puede resultar incómodo. El discurso puede tener resonancias esencialistas que no comparto. Es importante encontrar la forma de hablar de identidad sin caer en ninguna forma de esencialismo. Dejemos que Rich siga hablando:

Pensar como mujer en el mundo del hombre significa pensar críticamente, rehusar a aceptar lo dado, estableciendo conexiones entre hechos e ideas que los hombres han dejado desconectadas. Significa recordar que toda mente reside en un cuerpo y ser responsables de los cuerpos femeninos en los cuales vivimos, comprobando constantemente las hipótesis dadas frente a nuestra propia experiencia vivida. (Rich, 2010e: 357)

"Pensar como un hombre" ha sido un halago o una limitación para las mujeres que han querido escapar a la trampa del cuerpo. Para Rich (1986: 81), no es extraño que muchas mujeres inteligentes y creativas hayan insistido en que eran "seres humanos" primero y mujeres solo accidentalmente, y que esto las haya llevado a minimizar su biología y sus vínculos con otras mujeres. El cuerpo puede ser para la mujer una fuente de placer, pero también una prisión, puede ser una ocupación e incluso convertirse en una obsesión. Según Rich (1986: 401), el temor y el odio a nuestros cuerpos ha hecho que muchas mujeres enormemente inteligentes hayan tratado de pensar desde fuera de sus cuerpos:

No conozco a ninguna mujer - virgen, madre, lesbiana, casada, célibe, que gane su sustento como ama de casa, camarera o exploradora de las ondas cerebrales - para quien su cuerpo no sea el problema fundamental; su significado oscuro, su fertilidad, su deseo, su llamada frigidez, su sangre, sus silencios, sus cambios y mutilaciones, sus violaciones y maduraciones. (Rich, 1986: 390-401)

Ante lo problemático que resulta el cuerpo, muchas mujeres han preferido prescindir de él viajando como un espíritu incorpóreo (Rich, 1986: 81). En Nacemos de mujer, Rich afirma:

Al decir que de ningún modo hemos explorado o comprendido aún nuestro fundamento biológico ni el milagro o la paradoja del cuerpo femenino y sus significados político y espiritual, estoy preguntando si la mujer 
podrá comenzar de una vez para siempre a pensar con su cuerpo, y a relacionar todo aquello que tan cruelmente ha visto desorganizado: nuestras capacidades mentales, apenas utilizadas; nuestro sentido del tacto, tan desarrollado; nuestro talento para la observación aguda; nuestro organismo complicado y doloroso, y su placer mutilado. (Rich, 1986: 401; énfasis en el original)

Siguiendo la línea de Rich, de quien reconoce la deuda, Rosi Braidotti (2004: 40) afirma que la redefinición del sujeto feminista femenino empieza con la reevaluación de las raíces corporales de la subjetividad. Se trata de acabar con la visión antes tradicional del sujeto cognoscente como universal, neutro y consecuentemente desprovisto de género.

\section{La reivindicación de la experiencia propia}

En este punto no queremos dejar de citar el trabajo ya clásico de Joan Scott “The Evidence of Experience" (1991). No son los individuos los que tienen las experiencias, sino que son los sujetos los que son constituidos a través de las experiencias. Recordemos cómo interpreta Teresa de Lauretis la experiencia: "I use the term not in the individualistic, idiosyncratic sense of something belonging to one and exclusively her own even though others might have 'similar' experiences; but rather in the general sense of a process by which, for all social beings, subjectivity is constructed" (1984: 159).

La experiencia es el proceso por el cual se construye la subjetividad para todos los seres sociales. A través de ese proceso, uno se ubica o es ubicado en la realidad social y de ese modo percibe y comprende como subjetivas (referidas a uno mismo y originadas en él) las relaciones materiales, económicas e interpersonales, sin obviar en ningún caso la importancia del discurso, puesto que los sujetos son construidos discursivamente (Scott, 1991: 792-793). La experiencia vivida solo toma forma a través del relato. Dicho de otro modo, es a través de la narración que damos sentido a lo vivido.

Con políticas de localización, Rich apunta a que el pensamiento no es abstracto, universalizado, objetivo ni indiferente, sino que está situado en la contingencia de la propia experiencia y, como tal, es un ejercicio necesariamente parcial. La reivindicación de la experiencia propia trata, según Rich, de "reintegrar lo que se ha llamado inconsciente, subjetivo y emocional, en lo estructural, racional e intelectual". "La experiencia", dirá Rich, "nos moldea, al igual que el azar, las estrellas y el tiempo, nuestras propias adaptaciones y rebeliones, y, sobre todo, el orden social de nuestro alrededor" (1986: 19).

Frente al autor ausente al que no acaba de entender, ella escribe desde el testimonio personal que mezcla con la investigación y la teoría. Esa experiencia de la 
vida también es reivindicada por Rich. Experiencia y erudición se funden por ejemplo en Nacemos de mujer, donde mezcla su experiencia como madre primeriza, las conversaciones con sus hijos ya adultos o las charlas con amigas con interesantes análisis históricos y teóricos realizados sobre la maternidad.

Es preciso, pues, dar espacio a las experiencias femeninas a las que antes no se les había prestado suficiente atención. Se trataría de arraigar toda teoría en la experiencia personal y hablar desde nuestra propia experiencia. Según Benjamin (2017: 628), este interés por las experiencias de las mujeres podría en parte explicar su interés por registrar las vidas y la creación de archivos para guardar toda esta información. Gracias a ello, hoy podemos leer muchas de sus cartas. Según Rich, en cualquier disciplina donde se nos considere, las mujeres somos percibidas más como objeto que como sujetos de la investigación, y ello es así por tratarse de la óptica de los varones y por tratarnos a nosotras como una categoría especial (2010b: 195). Ellos explican nuestras experiencias. Ni siquiera el relato de la experiencia sexual es propio. Recojo dos citas de Rich donde se subraya cómo las experiencias sexuales de las mujeres son relatadas desde una perspectiva masculina. Así, "[a] la esposa victoriana o a la dama blanca sureña, de quienes se esperaba que no tuvieran sensualidad alguna, se les pedía quedarse 'impasibles': de la mujer 'liberada' del siglo veinte se espera que finja orgasmos" (2010c: 278), o "[1]a asexuada esposa angélica y la prostituta victorianas [...] no tenían nada que ver con la sensualidad real de las mujeres, y en cambio sí - y mucho- con la experiencia subjetiva que los hombres tienen de las mujeres" (1986: 73).

No hay una sola disciplina que no oscurezca y devalúe la historia y las experiencias de las mujeres. Las experiencias femeninas que continuamente se niegan, sin embargo, sí sirven para poner en cuestión las ideas feministas que tienden a ser presentadas como el resultado de un estallido personal de amargura. Frente a esto, Rich reivindica la experiencia femenina como algo valioso. Las mujeres deben poder explicar sus vidas desde sus experiencias y no a través del discurso de los otros. El énfasis en documentar la vida de las mujeres y el potencial que tiene este material para crear conexiones entre mujeres aparece para Rich como un método para dar forma a la comunidad feminista, así como nuevas formas de pensar cómo y de qué fuentes las feministas crean conocimiento (Benjamin, 2017: 629). Recordemos aquí a De Lauretis: "that political, theoretical, self analyzing practice by which the relations of the subjects in social reality can be rearticulated from the historical experience of women" (1984: 186). Como años después denunció Seyla Benhabib (1992: 13), la exclusión de las mujeres y de su punto de vista no solo es una omisión política y un punto ciego moral sino que también constituye un déficit epistemológico. 


\section{A modo de conclusión. De la experiencia propia a la experiencia compartida}

Lorraine Code (2000: xix) nos recuerda que las experiencias no hablan por sí mismas y de ahí que la toma de conciencia sea un componente vital para la práctica feminista, ya que ha ayudado a que las mujeres aprendan a reconocer, a nombrar y a compartir sus experiencias. Las mujeres que empiezan a hablar no son las mismas tras compartir los relatos y escuchar las experiencias de las otras. Esta toma de conciencia resulta fundamental para transformar la realidad. Recordemos la importancia que tuvieron los grupos de autoconciencia en tiempos del feminismo radical. Cito a Adrienne Rich:

Lo que las feministas radicales de hoy en día han llegado a admitir es que para poder llegar a ser una fuerza contra el elitismo y el exclusivismo debemos saber situarnos todas y cada una de nosotras, no para obstaculizar a cualquier otro ser humano, sino para apelar a las diferentes clases de poder y conocimiento que existen - enterradas, difusas, mal nombradas y a veces mal dirigidas- dentro de las mujeres. (2010b: 193-194)

Scott (1991: 787) tiene razón cuando advierte de la necesidad de ser cuidadosas cuando nos referimos a las experiencias de las mujeres, ya que sería erróneo obviar que en la creación de las subjetividades femeninas elementos como la raza o la sexualidad se intersectan con el género. Rich nos recuerda que lo social y lo personal están imbricados y que cambian con la historia, y las políticas de localización son muy sensibles a estas cuestiones. Recordemos, por ejemplo, como explica que cuando nació pesaba más en ella el hecho de ser blanca que el hecho de ser mujer o como la religión que profesaba su padre habría sido un elemento central de haber nacido en Centroeuropa. Ser judía se vuelve muy relevante con la llegada de Hitler al poder. La identidad se construye de un modo específico en ese momento, muy diferente a la forma como había sido construida antes y distinta a la forma como se construirá después. ${ }^{6}$ No podemos separar estas cuestiones de la historia. Las ubicaciones anteriores se pueden recordar y reconstruir de diferentes maneras y eso nos aleja de cualquier tipo de enfoque esencialista. No debemos pensar en la identidad como una producción ya finalizada sino, más bien, como algo siempre en proceso, algo que se está haciendo. Y deshaciendo. Y siempre en interacción con los otros.

\footnotetext{
${ }^{6}$ Considero que se podría establecer un paralelismo entre esto y el ejemplo que toma Scott de Stuart Hall acerca del negro jamaicano. Negro es una identidad que pudo ser aprendida en un momento específico (1991: 792).
}

302

Lectora, 27 (2021): 291-307. ISSN: 1136-5781 D.O.I.: 10.1344/Lectora2021.27.15 
Me gustaría concluir este trabajo planteando cómo podemos poner en común esas experiencias personales y cómo podemos construir un nosotras que aúne los múltiples yoes:

The issue of locatedness and the problem of the subject, that other major theoretical debate of recent years, intertwine and, in both Rich's work and politics of location writing generally, this double perspective is often expressed through what we might call the problem of pronouns - "I", the individual subject, "we", the collective subject and the relations between. The importance of saying "I" and "we" alongside the difficulty of saying "I" and "we" has been a refrain throughout the work of Rich: (Eagleton, 2000: 300)

El proyecto de cambiar las estructuras de la desigualdad se llevaría a cabo desde un conocimiento autoconsciente y analítico de la propia ubicación en las intersecciones de género, raza, clase, y orientación sexual (Rich, 2001: 153). Rich denunciará como en el feminismo radical "lo personal es político" acabó conduciendo a un "lo-personal-por-lo-personal-mismo" que desembocó en un "lo personal es bueno" olvidando todo lo que tenía que ver con lo colectivo. A Rich le preocupaba esta visión limitada de la experiencia que se centra en el yo olvidándose de las condiciones sociales y de las relaciones estructurantes que están por detrás y que se acaban obviando. No nos debemos quedar en los relatos en sí sin ser capaces de desvelar a partir de ellos el funcionamiento de determinados poderes para así poder iluminar las condiciones sociales de la existencia. Los relatos biográficos presentan un valor para el conocimiento y para la política en tanto que consiguen hablar de una historia, de un contexto, de relaciones sociales, etc. Si esto se pierde, el conocimiento que produce carece de valor epistémico y político real por lo que deja de tener sentido y nos lleva, además, a la peligrosa erradicación del "nosotras" como identidad feminista común y crítica social (Eagleton, 2000: 302). De este modo, las políticas de localización facilitarían la creación de nuevas formas de intersubjetividad (Fox, 2004: 42).

La historia del feminismo nos muestra una continua reelaboración del tema de la identidad. Están en juego múltiples identidades, en ocasiones complementarias, en ocasiones en competencia y, en ocasiones, también en conflicto. Recordemos que Rich (1982: 89) se presentaba como blanca, judía, antisemita, racista, antirracista, casada una vez con un hombre y lesbiana. Rich es consciente de estar involucrada en una negociación táctica a través de un laberinto de identidades (Eagleton, 2000: 305).

En un momento de crisis del sujeto del feminismo, en el núcleo de este concepto late el deseo de encontrar formas para establecer nexos profundos y 
responsables de unas con otras con el afán de poder provocar cambios sociales tanto a nivel individual como colectivo. La autoconciencia implica plantearnos quiénes somos y hacia dónde vamos. Son precisas unas políticas de la localización que permitan entender los relatos individuales y ser capaces de construir coaliciones y alianzas fuertes que permitan hacer más poderoso al feminismo en su lucha por lograr las reivindicaciones de las mujeres.

Tras nuestro análisis concluimos que las políticas de localización nos permiten centrarnos no solo en el sujeto sino también en el mundo. Frente a las identidades personales que nos separan, pensar los problemas desde los contextos que nos son comunes puede resultar interesante para, sorteando las heridas de las subjetividades, hallar un terreno de diálogo. Según Lober (2017: 668), entre los objetivos de las políticas de la localización y la forma de pensar la identidad que conlleva se halla un interés en fomentar potenciales coaliciones. Kaplan (1994: 139) plantea las políticas de localización, que parten del reconocimiento de las distintas experiencias de las mujeres, como una herramienta para lograr un feminismo transnacional capaz de crear una alianza mundial de mujeres. Ejemplo de ello puede ser cómo Rich defendió solidaridades anticoloniales, antirracistas y antiimperialistas que fueron el punto de partida para un feminismo solidario con Palestina y un antisionismo feminista judío capaz de cruzar fronteras y establecer una red transnacional de liberación (Lober, 2017: 667-668). Podemos decir, pues, que las políticas de la localización tienen un gran potencial ético que puede ayudar a vivir con los otros en un mundo heterogéneo (Lober, 2017: 681).

Según Perreault (1995: 44), la tensión que existe dentro de Rich entre sus múltiples facetas sería parecida a la tensión a la que nos enfrentaríamos cuando dialogamos o nos comparamos con otras mujeres. En ese sentido, ser conscientes del laberinto de identidades de nuestro interior podría ayudarnos a establecer diálogos con otras mujeres, insertas cada una de ellas en su maraña de opresiones y en sus identidades fragmentadas. Bridges: A Journal for Jewish Feminists and Our Friends se llamaba precisamente la revista dedicada a la causa del feminismo judío a la que Rich, desde 1990, dedicó buena parte de sus últimos años como coeditora. Esta revista tenía como meta poner en valor la multiplicidad de experiencias e identidades judías y, al mismo tiempo, se preocupó de establecer estos puentes también con otras tradiciones sacando a la luz, por ejemplo, voces latinoamericanas. Bridges, puentes, de eso se trata. Termino con unas palabras de Rich: "Hasta que entre madre e hija, entre mujer y mujer, a través de las generaciones, no se extienda una línea de amor, confirmación y ejemplo, las mujeres errarán siempre en el desierto" (1986: 353). 


\section{REFERENCIAS BIBLIOGRÁFICAS}

Anzaldúa, Gloria y Cherríe Moraga (eds.) (1981), This Bridge Called My Back: Writings by Radical Women of Color, Nueva York, Kitchen Table: Women of Color Press.

Benhabib, Seyla (1992), "Communicative Ethics and the Claims of Gender, Community and Postmodemism”, Situating the Self: Gender, Community, and Postmodernism in Contemporary Ethics, Nueva York, Routledge: 1-23.

Benjamin, Meredith (2017), "Snapshots of a Feminist Poet: Adrienne Rich and the Poetics of the Archive", Women's Studies, 46 (7): 628-645. <https://doi.org/10.1080/00497878.2017.1337415>

Braidotti, Rosi (2004), Feminismo, diferencia sexual y subjetividad nómade, Barcelona, Gedisa.

Butler, Judith (2007), El género en disputa, Madrid, Cátedra.

Code, Lorraine (ed.) (2000), Encyclopedia of Feminist Theories, Londres, Routledge.

Crenshaw, Kimberlé Williams (1989), "Demarginalizing the Intersection of Race and Sex: A Black Feminist Critique of Antidiscrimination Doctrine, Feminist Theory and Antiracist Politics", University of Chicago Legal Forum, 1: 139-168. <http://chicagounbound.uchicago.edu/uclf/vol1989/iss1/8>

De Lauretis, Teresa (1984), Alice Doesn't. Feminism, Semiotics, Cinema, Bloomington, Indiana UP.

-(1987), Technologies of Gender: Essays on Theory, Film and Fiction, Bloomington, Indiana UP.

Eagleton, Mary (2000), “Adrienne Rich, Location and the Body”, Journal of Gender Studies, 9 (3): 299-312.

Falcón, Sylvanna M. (2012), “Transnational Feminism and Contextualized Intersectionality at the 2001 World Conference Against Racism", Journal of Women's History, 24 (4): 99-120.

Fox, Catherine O. M. (2004), "Be-coming Subjects: Reclaiming a Politics of Location as Radical Political Rhetoric", Retrospective Theses and Dissertations, 775. <https://lib.dr.iastate.edu/rtd/775>

Haraway, Donna (1988), "Situated Knowledges: The Science Question in Feminism and the Privilege of Partial Perspective", Feminist Studies, 14 (3): 575-599.

Hull, Gloria T.; Patricia Bell Scott y Barbara Smith (eds.) (1982), All the Women Are White, All the Men Are Black, But Some of Us Are Brave: Black Women's Studies, Old Westbury NY, The Feminist Press. 
Kaplan, Caren (1994), “The Politics of Location as Transnational Feminist Practice", Scattered Hegemonies: Postmodernity and Transnational Feminist Practices, Inderpal Grewal y Caren Kaplan (eds.), Minneapolis, University of Minnesota Press: 137-152.

Lober, Brooke (2017), “Adrienne Rich's 'Politics of Location', US Jewish Feminism, and the Question of Palestine”, Women's Studies, 46 (7): 663-683.

Mohanty, Chandra Talpade (1987), "Feminist Encounters: Locating the Politics of Experience", Copyright, 1: 30-44.

Mouffe, Chantal (1999), El retorno de lo político, Marco Aurelio Galbarini (trad.), Barcelona, Paidós.

Papic, Zarana (1994), "Nationalism, Patriarchy and War in Ex-Yugoslavia”, Women's History Review, 3 (1): 115-117.

Perreault, Jeanne (1995), Writing Selves: Contemporary Feminist Autography, Minneapolis, University of Minnesota Press.

Rich, Adrienne (1972), “When We Dead Awaken: Writing as Re-Vision”, College English, 34 (1): 18-30.

-(1982), "Split at the Root", Nice Jewish Girls: A Lesbian Feminist Anthology, Evelyn Torton Beck (ed.), Boston, Beacon Press: 73-90.

-(1983), "Blood, Bread and Poetry. The Location of the Poet", The Massachusetts Review, Autumn, 24 (3): 521-540.

-(1986), Nacemos de mujer. La maternidad como experiencia e institución, Madrid, Cátedra Feminismos.

-(1994a), "Notes towards a Politics of Location", Blood, Bread and Poetry. Selected Prose 1979-1985, Londres, Virago Press: 210-232.

—(1994b), "Invisibility in Academe”, Blood, Bread and Poetry. Selected Prose 19791985, Londres, Virago Press: 198-201.

-(2001), Arts of the Possible: Essays and Conversations. Nueva York, Norton.

-(2010a), "Enseñar estudiantes por "libre", Sobre mentiras, secretos y silencios, Madrid, Horas y HORAS: 73-100.

—(2010b), "La mujer antifeminista", Sobre mentiras, secretos y silencios, Madrid, Horas y HORAS: 101-123.

—(2010c), "Mujeres y honor: Algunas notas sobre el mentir", Sobre mentiras, secretos y silencios, Madrid, Horas y HORAS: 273-284.

_-(2010d), "Es la lesbiana que hay en nosotras", Sobre mentiras, secretos y silencios, Madrid, Horas y HORAS: 289-294.

-(2010e), "Hay que tomar en serio los estudios de la mujer", Sobre mentiras, secretos y silencios, Madrid, Horas y HORAS: 345-357. 
Scott, Joan (1991), “The Evidence of Experience”, Critical Inquiry, 17 (4): 773-797. Woolf, Virginia (1993), Three Guineas, Londres, Penguin. [1938]

\section{(c) (1) $(9)$}

